\title{
Supersymmetric Double Field Theory: Stringy Reformulation of Supergravity
}

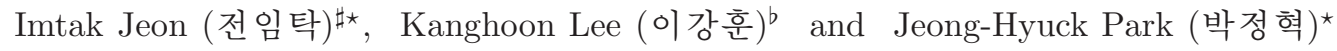 \\ ${ }^{\sharp}$ CERN, Theory Division CH-1211 Geneva 23, Switzerland \\ *Department of Physics, Sogang University, Seoul 121-742, Korea \\ ${ }^{b}$ Center for Quantum Spacetime, Sogang University, Seoul 121-742, Korea \\ imtak@sogang.ac.kr, kanghoon@sogang.ac.kr, park@sogang.ac.kr
}

\begin{abstract}
We construct a supersymmetric extension of double field theory that realizes the ten-dimensional Majorana-Weyl local supersymmetry. In terms of a stringy differential geometry we proposed earlier, our action consists of five simple terms - two bosonic plus three fermionic - and manifests not only diffeomorphism and one-form gauge symmetry of $B$-field, but also $\mathbf{O}(10,10)$ T-duality as well as a direct product of two local Lorentz symmetries, $\mathbf{S O}(1,9) \times \mathbf{S O}(9,1)$. A gauge fixing that identifies the double local Lorentz groups reduces our action to the minimal supergravity in ten dimensions.

PACS numbers: 04.60.Cf, 04.65.+e
\end{abstract}

Without resorting to vector notation, Maxwell's original equations consisted of twenty formulas. It was the rotational or Lorentz symmetry that reorganized them into four or two compact equations. Recent developments in string theory indicate that supergravity theories - at least those which have stringy origin - may undergo a similar reformulation, and be greatly simplified with the renewed understanding of their stringy structure or T-duality.

T-duality is a genuine stringy effect such that string theory effective actions or ten-dimensional supergravities should feature $\mathbf{O}(10,10)$ structure $[1[4]$. The $\mathbf{O}(10,10)$ T-duality can be manifestly realized if we formally double the spacetime dimension, from ten to twenty with coordinates $x^{\mu} \rightarrow y^{A}=\left(\tilde{x}_{\mu}, x^{\nu}\right)$ [5] [8], and reformulate the ten-dimensional effective action in terms of twenty dimensional language i.e. tensors equipped with $\mathbf{O}(10,10)$ metric,

$$
\mathcal{J}_{A B}=\left(\begin{array}{ll}
0 & 1 \\
1 & 0
\end{array}\right)
$$

This kind of reformulation was coined Double Field Theory (DFT) 9 12 , and has attracted much attention in recent years [13 31]. In DFT, as a field theory counterpart to the level matching condition of closed string theories, the $\mathbf{O}(10,10)$ d'Alembertian operator must be trivial, acting on arbitrary fields as well as their products,

$$
\partial^{2} \Phi=\partial_{A} \partial^{A} \Phi \equiv 0, \quad \partial_{A} \Phi_{1} \partial^{A} \Phi_{2} \equiv 0 .
$$

Hence locally, up to $\mathbf{O}(10,10)$ rotation, all the fields are independent of the dual coordinates, $\frac{\partial}{\partial \tilde{x}_{\mu}} \equiv 0$, and the theory is not truly doubled [11].

In a sense, the $\mathbf{O}(10,10)$ structure in DFT is a "metasymmetry" rather than a Noether symmetry, since only after dimensional reductions can it generate a Noether symmetry. Another feature of DFT is that, the diffeomorphism and the one-form gauge symmetry of $B$-field are naturally unified into what we may call "double-gauge symmetry," as they are generated by the generalized Lie derivative [8, 12, 32 34,

$$
\begin{aligned}
& \hat{\mathcal{L}}_{X} T_{A_{1} \cdots A_{n}}:=X^{B} \partial_{B} T_{A_{1} \cdots A_{n}}+\omega_{T} \partial_{B} X^{B} T_{A_{1} \cdots A_{n}} \\
& \quad+\sum_{i=1}^{n}\left(\partial_{A_{i}} X_{B}-\partial_{B} X_{A_{i}}\right) T_{A_{1} \cdots A_{i-1}}{ }^{B}{ }_{A_{i+1} \cdots A_{n}}
\end{aligned}
$$

where $\omega_{T}$ is the weight of $T_{A_{1} \cdots A_{n}}$. Since this differs from the ordinary Lie derivative, the underlying differential geometry of DFT is not Riemannian [7, 8, 14 16, 18, 32 37 (see [38, 39] for extensions to $\mathcal{M}$-theory). Namely, while doubling the spacetime dimension is sufficient to manifest the $\mathbf{O}(10,10)$ structure, the double-gauge symmetry (3) calls for novel mathematical treatment.

In this paper, we construct a supersymmetric extension of double field theory that manifests simultaneously $\mathbf{O}(10,10)$ T-duality and various gauge symmetries listed in Table凹including the double-gauge symmetry. In order to do so, we employ the stringy differential geometry we developed earlier in Refs. 14 16]. Especially we utilize the "semi-covariant" derivatives proposed therein.

- $\mathbf{O}(10,10)$ T-duality: Meta-symmetry

- Gauge symmetries

1. Double-gauge symmetry

- Diffeomorphism

- One-form gauge symmetry

2. Local Lorentz symmetries, $\mathbf{S O}(1,9) \times \mathbf{S O}(9,1)$

3. Local Majorana-Weyl supersymmetry

TABLE I. T-duality and gauge symmetries in super DFT.

The supersymmetric DFT action we construct below, Eq.(28), reformulates the ten-dimensional minimal, i.e. $\mathcal{N}=1$ supergravity into five simple terms, while doubling the local Lorentz symmetries. For a related superspace analysis we refer readers to an earlier work by Siegel [8]. 


\section{SETUP}

Our supersymmetric extension is minimal such that the field contents include the DFT-dilaton, doublevielbeins, DFT-dilatino and gravitino, as well as a local supersymmetry parameter,

$$
d, \quad V_{A p}, \quad \bar{V}_{B \bar{q}}, \quad \rho^{\alpha}, \quad \psi_{\bar{p}}^{\alpha}, \quad \varepsilon^{\alpha} .
$$

Their weights are trivial except the DFT-dilaton, $d$, as it is related to the conventional string dilaton, $\phi$, through $e^{-2 d}=\sqrt{-g} e^{-2 \phi}[11]$, such that $e^{-2 d}$ has weight unity and

$$
\hat{\mathcal{L}}_{X} d:=-\frac{1}{2} e^{2 d} \hat{\mathcal{L}}_{X}\left(e^{-2 d}\right)=X^{A} \partial_{A} d-\frac{1}{2} \partial_{A} X^{A} .
$$

Every field in (4) is covariant under all the bosonic symmetries in Table [ The indices of each field denote the relevant symmetry representations, as summarized in Table II]

\begin{tabular}{||c|c|c||}
\hline Index & Representation & Metric \\
\hline$A, B, \cdots$ & $\left.\begin{array}{c}\mathbf{O}(10,10) \\
\text { double-gauge }\end{array}\right\}$ vector & $\mathcal{J}_{A B}$ in Eq.(11) \\
$p, q, \cdots$ & $\mathbf{S O}(1,9)$ vector & $\eta_{p q}=\operatorname{diag}(-++\cdots+)$ \\
$\bar{p}, \bar{q}, \cdots$ & $\mathbf{S O}(9,1)$ vector & $\bar{\eta}_{\bar{p} \bar{q}}=\operatorname{diag}(+--\cdots-)$ \\
$\alpha, \beta, \cdots$ & $\mathbf{S p i n}(1,9)$ spinor & $C_{\alpha \beta}$ in Eq.([6) \\
\hline
\end{tabular}

TABLE II. Indices for each symmetry representation and the relevant metrics that raise or lower the positions of them.

With the real $\mathbf{S O}(1,9)$ gamma matrices, $\left(\gamma^{p}\right)^{\alpha}{ }_{\beta}$, the charge conjugation matrix, $C_{\alpha \beta}$, satisfies

$$
\left(C \gamma^{p_{1} p_{2} \cdots p_{n}}\right)_{\alpha \beta}=-(-1)^{n(n+1) / 2}\left(C \gamma^{p_{1} p_{2} \cdots p_{n}}\right)_{\beta \alpha},
$$

and defines the conjugated spinors, $\bar{\psi}_{\bar{p} \alpha}=\psi_{\bar{p}}^{\beta} C_{\beta \alpha}, \bar{\rho}_{\alpha}=$ $\rho^{\beta} C_{\beta \alpha}$, etc. All the spinors are taken to be $\mathbf{O}(10,10)$ singlet and Majorana-Weyl, possessing definite chiralities. With $\gamma^{(10)}=\gamma^{012 \cdots 9}$ they obey

$$
\gamma^{(10)} \psi_{\bar{p}}=+\psi_{\bar{p}}, \quad \gamma^{(10)} \rho=-\rho, \quad \gamma^{(10)} \varepsilon=+\varepsilon .
$$

The double-vielbein satisfies the defining properties [15],

$$
\begin{array}{rlrl}
V_{A p} V_{q}^{A} & =\eta_{p q}, & & V_{A p} \bar{V}_{\bar{q}}^{A}=0, \\
\bar{V}_{A \bar{p}} \bar{V}_{\bar{q}}^{A}=\bar{\eta}_{\bar{p} \bar{q}}, & & V_{A p} V_{B}^{p}+\bar{V}_{A \bar{p}} \bar{V}_{B}^{\bar{p}}=\mathcal{J}_{A B} .
\end{array}
$$

Hence it generates a pair of rank-two projections [14],

$$
P_{A B}:=V_{A}^{p} V_{B p}, \quad \bar{P}_{A B}:=\bar{V}_{A}^{\bar{p}} \bar{V}_{B \bar{p}},
$$

that are symmetric, orthogonal and complementary, as

$$
\begin{array}{ll}
P_{A B}=P_{B A}, & \bar{P}_{A B}=\bar{P}_{B A}, \\
P_{A}{ }^{B} P_{B}{ }^{C}=P_{A} C, & \bar{P}_{A}^{B} \bar{P}_{B}{ }^{C}=\bar{P}_{A}{ }^{C}, \\
P_{A}{ }^{B} \bar{P}_{B}^{C}=0, & P_{A}^{B}+\bar{P}_{A}^{B}=\delta_{A}^{B} .
\end{array}
$$

Further they give a pair of rank-six, symmetric and traceless projections [15],

$$
\begin{aligned}
& \mathcal{P}_{C A B}{ }^{D E F}:=P_{C}{ }^{D} P_{[A}{ }^{[E} P_{B]}{ }^{F]}+\frac{2}{9} P_{C[A} P_{B]}{ }^{[E} P^{F] D}, \\
& \overline{\mathcal{P}}_{C A B}{ }^{D E F}:=\bar{P}_{C}{ }^{D} \bar{P}_{[A}{ }^{[E} \bar{P}_{B]}{ }^{F]}+\frac{2}{9} \bar{P}_{C[A} \bar{P}_{B]}{ }^{[E} \bar{P}^{F] D} .
\end{aligned}
$$

We are now ready to recall the three differential operators from [14 16] and generalize them to include 'torsion',

$$
\begin{aligned}
& \nabla_{A}=\partial_{A}+\Gamma_{A}, \\
& D_{A}=\partial_{A}+\Phi_{A}+\bar{\Phi}_{A}, \\
& \mathcal{D}_{A}=\partial_{A}+\Gamma_{A}+\Phi_{A}+\bar{\Phi}_{A} .
\end{aligned}
$$

The first, $\nabla_{A}$, is the semi-covariant derivative for the double-gauge symmetry we developed in [14, 15],

$$
\begin{aligned}
\nabla_{C} T_{A_{1} A_{2} \cdots A_{n}}:= & \partial_{C} T_{A_{1} A_{2} \cdots A_{n}}-\omega_{T} \Gamma^{B}{ }_{B C} T_{A_{1} A_{2} \cdots A_{n}} \\
& +\sum_{i=1}^{n} \Gamma_{C A_{i}}{ }^{B} T_{A_{1} \cdots A_{i-1}} B A_{i+1} \cdots A_{n}
\end{aligned} .
$$

The second, $D_{A}$, is a covariant derivative for the pair of local Lorenz symmetries having the connections, $\Phi_{A}$ and $\bar{\Phi}_{A}$ for $\mathbf{S O}(1,9)$ and $\mathbf{S O}(9,1)$ respectively [16].

The last, $\mathcal{D}_{A}$, is the "master" derivative combining $\nabla_{A}$ and $D_{A}$ [16]. As for the unifying description of the closed string massless bosonic sector, it annihilates all the bosonic fields in (4),

$$
\begin{aligned}
& \mathcal{D}_{A} V_{B p}=\partial_{A} V_{B p}+\Gamma_{A B}{ }^{C} V_{C p}+\Phi_{A p}{ }^{q} V_{B q}=0, \\
& \mathcal{D}_{A} \bar{V}_{B \bar{p}}=\partial_{A} \bar{V}_{B \bar{p}}+\Gamma_{A B}{ }^{C} \bar{V}_{C \bar{p}}+\bar{\Phi}_{A \bar{p}} \bar{q} \bar{V}_{B \bar{q}}=0, \\
& \mathcal{D}_{A} d:=-\frac{1}{2} e^{2 d} \nabla_{A}\left(e^{-2 d}\right)=\partial_{A} d+\frac{1}{2} \Gamma^{B}{ }_{B A}=0,
\end{aligned}
$$

and also all the "constants," $\mathcal{J}_{A B}, \eta_{p q}, \bar{\eta}_{\bar{p} \bar{q}}, C_{\alpha \beta},\left(\gamma^{p}\right)^{\alpha}{ }_{\beta}$. The connections are all skew-symmetric and related to each other, from (8), (14), through

$$
\begin{aligned}
& \Phi_{A p q}=-\Phi_{A q p}=V_{p}^{B} \nabla_{A} V_{B q}, \\
& \bar{\Phi}_{A \bar{p} \bar{q}}=-\bar{\Phi}_{A \bar{q} \bar{p}}=\bar{V}_{\bar{p}}^{B} \nabla_{A} \bar{V}_{B \bar{q}}, \\
& \Gamma_{A B C}=-\Gamma_{A C B}=V_{B}{ }^{p} D_{A} V_{C p}+\bar{V}_{B} \bar{p} D_{A} \bar{V}_{C \bar{p}},
\end{aligned}
$$

such that they assume the following most general forms,

$$
\begin{aligned}
& \Phi_{A p q}=\Phi_{A p q}^{0}+\Delta_{A p q}, \quad \bar{\Phi}_{A \bar{p} \bar{q}}=\bar{\Phi}_{A \bar{p} \bar{q}}^{0}+\bar{\Delta}_{A \bar{p} \bar{q}}, \\
& \Gamma_{C A B}=\Gamma_{C A B}^{0}+\Delta_{C p q} V_{A}^{p} V_{B}^{q}+\bar{\Delta}_{C \bar{p} \bar{q}} \bar{V}_{A} \bar{p} \bar{V}_{B} \bar{q}
\end{aligned}
$$

Here, from [15],

$$
\begin{aligned}
& \Gamma_{C A B}^{0}=2\left(P \partial_{C} P \bar{P}\right)_{[A B]} \\
& \quad+2\left(\bar{P}_{[A}{ }^{D} \bar{P}_{B]}^{E}-P_{[A}{ }^{D} P_{B]}{ }^{E}\right) \partial_{D} P_{E C} \\
& \quad-\frac{4}{9}\left(\bar{P}_{C[A} \bar{P}_{B]}^{D}+P_{C[A} P_{B]}{ }^{D}\right)\left(\partial_{D} d+\left(P \partial^{E} P \bar{P}\right)_{[E D]}\right),
\end{aligned}
$$

and, with the corresponding derivative, $\nabla_{A}^{0}=\partial_{A}+\Gamma_{A}^{0}$,

$$
\Phi_{A p q}^{0}=V_{p}^{B} \nabla_{A}^{0} V_{B q}, \quad \bar{\Phi}_{A \bar{p} \bar{q}}^{0}=\bar{V}^{B}{ }_{\bar{p}} \nabla_{A}^{0} \bar{V}_{B \bar{q}} .
$$

As $\Gamma_{A B C}^{0}=\Gamma_{A[B C]}^{0}$ in (17) is the unique connection that further obeys [15, 16],

$$
\Gamma_{[A B C]}^{0}=0, \quad(\mathcal{P}+\overline{\mathcal{P}})_{C A B}{ }^{D E F} \Gamma_{D E F}^{0}=0,
$$


$\Gamma_{A}^{0}, \quad \Phi_{B}^{0}$ and $\bar{\Phi}_{C}^{0}$ correspond to the "minimal" or "torsionless" connections. The extra covariant pieces, $\Delta_{A p q}=-\Delta_{A q p}$ and $\bar{\Delta}_{A \bar{p} \bar{q}}=-\bar{\Delta}_{A \bar{q} \bar{p}}$, then can be viewed as torsion [16], being subject to

$$
\Delta_{A p q} V^{A p}=0, \quad \bar{\Delta}_{A \bar{p} \bar{q}} \bar{V}^{A \bar{p}}=0,
$$

which are necessary to maintain $\mathcal{D}_{A} d=0$. As is the case in ordinary supergravities, the torsion can be constructed from the bi-spinorial objects. Specifically, in the present work we set

$$
\begin{aligned}
\Gamma_{A B C}= & \Gamma_{A B C}^{0}+i \frac{1}{3} \bar{\rho} \gamma_{A B C} \rho+i \frac{1}{3} \bar{\psi} \bar{p} \gamma_{A B C} \psi_{\bar{p}} \\
& -2 i \bar{\rho} \gamma_{B C} \psi_{A}-4 i \bar{\psi}_{B} \gamma_{A} \psi_{C} .
\end{aligned}
$$

Further, hereafter, for simplicity we put

$$
\begin{array}{ll}
\psi_{A}:=\bar{V}_{A}{ }^{\bar{p}} \psi_{\bar{p}}, & \gamma^{A}:=V^{A}{ }_{p} \gamma^{p}, \\
\mathcal{D}_{p}:=V^{A}{ }_{p} \mathcal{D}_{A}, & \mathcal{D}_{\bar{p}}:=\bar{V}^{A}{ }_{\bar{p}} \mathcal{D}_{A},
\end{array}
$$

such that $\bar{V}_{\bar{p}}^{A} \psi_{A}=\psi_{\bar{p}}$ and $\left\{\gamma^{A}, \gamma^{B}\right\}=2 P^{A B}$, etc.

From $\left[\mathcal{D}_{A}, \mathcal{D}_{B}\right] V_{C p}=0,\left[\mathcal{D}_{A}, \mathcal{D}_{B}\right] \bar{V}_{C \bar{p}}=0$, the usual curvatures of the three connections, $\Gamma_{A}, \Phi_{A}, \bar{\Phi}_{A}$,

$$
\begin{aligned}
& R_{C D A B}=\partial_{A} \Gamma_{B C D}+\Gamma_{A C}{ }^{E} \Gamma_{B E D}-(A \leftrightarrow B), \\
& F_{A B p q}=\partial_{A} \Phi_{B p q}-\partial_{B} \Phi_{A p q}+\Phi_{A p r} \Phi_{B}^{r} q-\Phi_{B p r} \Phi_{A}^{r} q, \\
& \bar{F}_{A B \bar{p} \bar{q}}=\partial_{A} \bar{\Phi}_{B \bar{p} \bar{q}}-\partial_{B} \bar{\Phi}_{A \bar{p} \bar{q}}+\bar{\Phi}_{A \bar{p} \bar{r}} \bar{\Phi}_{B}{ }^{\bar{r}} \bar{q}-\bar{\Phi}_{B \bar{p} \bar{r}} \bar{\Phi}_{A}^{\bar{r}} \bar{q},
\end{aligned}
$$

satisfy the following relation,

$$
R_{A B C D}=F_{C D p q} V_{A}^{p} V_{B}^{q}+\bar{F}_{C D \bar{p} \bar{q}} \bar{V}_{A} \bar{p}^{\bar{q}} \bar{V}_{B}^{\bar{q}} .
$$

However, they are not double-gauge covariant 15]. Covariant quantities are achievable if we define [15]

$$
S_{A B C D}:=\frac{1}{2}\left(R_{A B C D}+R_{C D A B}-\Gamma_{A B}^{E} \Gamma_{E C D}\right),
$$

satisfying, with $\Gamma_{A B C}=\Gamma_{A B C}^{0}+\Lambda_{A B C}$ (16), (17),

$$
\begin{aligned}
& S_{A B C D}=S_{A B C D}^{0}+\mathcal{D}_{[A}^{0} \Lambda_{B] C D}+\mathcal{D}_{[C}^{0} \Lambda_{D] A B} \\
& \quad+\Lambda_{D[A}{ }^{E} \Lambda_{|C| B] E}+\Lambda_{B[C}{ }^{E} \Lambda_{|A| D] E}-\frac{1}{2} \Lambda^{E}{ }_{A B} \Lambda_{E C D}
\end{aligned}
$$

Examples of the covariant quantities include

$$
\begin{gathered}
P^{A B} P^{C D} S_{A C B D}, \quad \bar{P}^{A B} \bar{P}^{C D} S_{A C B D}, \\
S_{p \bar{q}}+2 i \bar{\psi}^{A} \gamma_{p} \mathcal{D}_{A} \psi_{\bar{q}}-2 i \bar{\psi}_{\bar{q}} \mathcal{D}_{p} \rho, \\
\gamma^{A} \mathcal{D}_{A} \rho, \quad \gamma^{A} \mathcal{D}_{A} \psi_{\bar{p}}, \quad \mathcal{D}_{\bar{p}} \rho, \quad \mathcal{D}_{A} \psi^{A}, \\
\bar{\psi}^{A} \gamma_{p}\left(\mathcal{D}_{A} \psi_{\bar{q}}-\frac{1}{2} \mathcal{D}_{\bar{q}} \psi_{A}\right),
\end{gathered}
$$

where $S_{p \bar{q}}=V^{A}{ }_{p} \bar{V}^{B}{ }_{\bar{q}} S_{A B}$ and $S_{A B}=S_{A C B}{ }^{C}$. This generalizes our earlier results [15, 16] to the torsionful connection (21).

\section{SUPERSYMMETRIC DFT LAGRANGIAN}

The supersymmetric double field theory Lagrangian we construct in this work consists of five terms ( $c f$. [8]):

$$
\begin{aligned}
\mathcal{L}_{\mathrm{SDFT}}= & e^{-2 d}\left[\frac{1}{8}\left(P^{A B} P^{C D}-\bar{P}^{A B} \bar{P}^{C D}\right) S_{A C B D}\right. \\
& \left.+i \frac{1}{2} \bar{\rho} \gamma^{A} \mathcal{D}_{A}^{\star} \rho+i \bar{\psi}^{A} \mathcal{D}_{A}^{\star} \rho+i \frac{1}{2} \bar{\psi}^{B} \gamma^{A} \mathcal{D}_{A}^{\star} \psi_{B}\right],
\end{aligned}
$$

where, with (21), $\mathcal{D}_{A}^{\star}$ is defined by its own connection,

$$
\begin{aligned}
\Gamma_{A B C}^{\star}= & \Gamma_{A B C}-i \frac{11}{96} \bar{\rho} \gamma_{A B C} \rho-i \frac{5}{24} \bar{\psi} \bar{p} \gamma_{A B C} \psi_{\bar{p}} \\
& +i \frac{5}{4} \bar{\rho} \gamma_{B C} \psi_{A}+2 i \bar{\psi}_{B} \gamma_{A} \psi_{C} .
\end{aligned}
$$

From (27) and [15, 16], each term in the Lagrangian is invariant under all the bosonic symmetries listed in Table I. while the whole Lagrangian is supersymmetric, up to the strong level matching constraint (2), under

$$
\begin{aligned}
\delta_{\varepsilon} d & =i \frac{1}{2} \bar{\varepsilon} \rho, \\
\delta_{\varepsilon} V_{A p} & =i \bar{\varepsilon} \gamma_{p} \psi_{A}, \\
\delta_{\varepsilon} \bar{V}_{A \bar{p}} & =-i \bar{\varepsilon} \gamma_{A} \psi_{\bar{p}}, \\
\delta_{\varepsilon} \rho & =-\gamma^{A} \hat{\mathcal{D}}_{A} \varepsilon, \\
\delta_{\varepsilon} \psi_{\bar{p}} & =\bar{V}^{A}{ }_{\bar{p}} \hat{\mathcal{D}}_{A} \varepsilon-i \frac{1}{4}\left(\bar{\rho} \psi_{\bar{p}}\right) \varepsilon-i \frac{1}{2}(\bar{\varepsilon} \rho) \psi_{\bar{p}},
\end{aligned}
$$

where, again with (21), $\hat{\mathcal{D}}_{A}$ is set by another connection,

$\hat{\Gamma}_{A B C}=\Gamma_{A B C}-i \frac{17}{48} \bar{\rho} \gamma_{A B C} \rho-i \frac{1}{4} \bar{\psi} \bar{p} \gamma_{A B C} \psi_{\bar{p}}+i \frac{5}{2} \bar{\rho} \gamma_{B C} \psi_{A}$

Under arbitrary variations of all the fields, we get

$$
\begin{aligned}
& \delta P_{A B}=-\delta \bar{P}_{A B}=2 \delta V_{(A}{ }^{p} V_{B) p}, \\
& \delta V_{A p}=\delta V_{B p} \bar{P}^{B}{ }_{A}+\delta V_{B[p} V^{B}{ }_{q]} V_{A}^{q}, \\
& \delta \psi_{A}=\left(\delta \psi_{\bar{p}}+\psi_{\bar{q}} \delta \bar{V}_{B} \bar{q}^{\bar{q}} \bar{V}_{\bar{p}}\right) \bar{V}_{A}^{\bar{p}}-\psi_{B} \delta V^{B}{ }_{p} V_{A}{ }^{p}, \\
& \delta \Phi_{A p q}=\mathcal{D}_{A}\left(V^{B}{ }_{p} \delta V_{B q}\right)+V^{B}{ }_{p} V^{C}{ }_{q} \delta \Gamma_{A B C}, \\
& \delta \bar{\Phi}_{A \bar{p} \bar{q}}=\mathcal{D}_{A}\left(\bar{V}^{B}{ }_{\bar{p}} \delta \bar{V}_{B \bar{q}}\right)+\bar{V}^{B}{ }_{\bar{p}} \bar{V}^{C}{ }_{\bar{q}} \delta \Gamma_{A B C}, \\
& \delta S_{A B C D}=\mathcal{D}_{[A} \delta \Gamma_{B] C D}-\frac{3}{2} \Gamma_{[E A B]} \delta \Gamma_{C D}^{E}+[(A, B) \leftrightarrow(C, D)],
\end{aligned}
$$

and, the Lagrangian transforms up to total derivatives $(\cong)$ as

$$
\begin{aligned}
\delta \mathcal{L}_{\mathrm{SDFT}} \cong & -2 \delta d \times \mathcal{L}_{\mathrm{SDFT}}+\delta \Gamma_{A B C} \times 0 \\
+ & \frac{1}{2} e^{-2 d} \delta V^{B p} \bar{V}_{B} \bar{q} \\
& \times\left(S_{p \bar{q}}-2 i \bar{\psi}_{\bar{q}} \hat{\mathcal{D}}_{p} \rho+i \bar{\psi}^{A} \gamma_{p} \mathcal{D}_{\bar{q}}^{b} \psi_{A}+i \bar{\rho} \gamma_{p} \mathcal{D}_{\bar{q}}^{\sharp} \rho\right) \\
+ & i e^{-2 d}\left(\delta \bar{\rho}-\frac{1}{4} \delta V_{B q} \bar{\rho} \gamma^{B q}\right) \times\left(\gamma^{A} \mathcal{D}_{A}^{\sharp} \rho-\hat{\mathcal{D}}_{A} \psi^{A}\right) \\
+ & i e^{-2 d}\left(\delta \bar{\psi}^{\bar{p}}+\bar{\psi}^{\bar{q}} \delta \bar{V}_{B \bar{q}} \bar{V}^{B \bar{p}}-\frac{1}{4} \delta V_{B q} \bar{\psi}^{\bar{p}} \gamma^{B q}\right) \\
& \times\left(\hat{\mathcal{D}}_{\bar{p}} \rho+\gamma^{A} \mathcal{D}_{A}^{b} \psi_{\bar{p}}\right) .
\end{aligned}
$$

From this, covariant, four sorts of equations of motion (two bosonic and two fermionic) can be readily read off. Here we let for $\mathcal{D}_{A}^{\sharp}, \mathcal{D}_{A}^{b}$ in the fermionic equations of motion,

$$
\begin{aligned}
\Gamma_{A B C}^{\sharp}= & \Gamma_{A B C}-i \frac{31}{96} \bar{\psi}^{\bar{r}} \gamma_{A B C} \psi_{\bar{r}}+i \frac{17}{24} \bar{\rho} \gamma_{B C} \psi_{A}, \\
\Gamma_{A B C}^{b}= & \Gamma_{A B C}-i \frac{31}{96} \bar{\rho} \gamma_{A B C} \rho-i \frac{5}{12} \overline{\psi^{\bar{r}}} \gamma_{A B C} \psi_{\bar{r}} \\
& +i \frac{1}{2} \bar{\rho} \gamma_{B C} \psi_{A}+4 i \bar{\psi}_{B} \gamma_{A} \psi_{C} .
\end{aligned}
$$

The 1.5 formalism that is familiar in ordinary supergravities holds in (33): the variation of the Lagrangian by $\delta \Gamma_{A B C}$, with (32), identically vanishes for the solution (21), while the equations of motion for the fermions can be obtained from the fermionic sector only i.e. the last three terms in the Lagrangian (28), with (29). 


\section{DFT SUPERSYMMETRY ALGEBRA}

Starting from the supersymmetry transformation rule of each DFT field in Eq.(30), through straightforward yet somewhat lengthy computations, we can obtain the following supersymmetry commutator relations, up to the strong level matching constraint (2),

$$
\begin{aligned}
& {\left[\delta_{\varepsilon_{1}}, \delta_{\varepsilon_{2}}\right] d \equiv \hat{\mathcal{L}}_{X_{3}} d,} \\
& {\left[\delta_{\varepsilon_{1}}, \delta_{\varepsilon_{2}}\right] V_{A p} \equiv \hat{\mathcal{L}}_{X_{3}} V_{A p}+\delta_{\varepsilon_{3}} V_{A p}+\Lambda_{p q} V_{A}^{q},} \\
& {\left[\delta_{\varepsilon_{1}}, \delta_{\varepsilon_{2}}\right] \bar{V}_{A \bar{p}} \equiv \hat{\mathcal{L}}_{X_{3}} \bar{V}_{A \bar{p}}+\delta_{\varepsilon_{3}} \bar{V}_{A \bar{p}}+\bar{\Lambda}_{\bar{p} \bar{q}} \bar{V}_{A}^{\bar{q}},} \\
& {\left[\delta_{\varepsilon_{1}}, \delta_{\varepsilon_{2}}\right] \rho \equiv \hat{\mathcal{L}}_{X_{3}} \rho+\delta_{\varepsilon_{3}} \rho+\frac{1}{4} \Lambda_{p q} \gamma^{p q} \rho} \\
& \quad-\frac{1}{2} X_{3}^{p} \gamma_{p}\left(\gamma^{A} \mathcal{D}_{A}^{\sharp} \rho-\hat{\mathcal{D}}_{A} \psi^{A}\right), \\
& {\left[\delta_{\varepsilon_{1}}, \delta_{\varepsilon_{2}}\right] \psi_{\bar{p}} \equiv \hat{\mathcal{L}}_{X_{3}} \psi_{\bar{p}}+\delta_{\varepsilon_{3}} \psi_{\bar{p}}+\frac{1}{4} \Lambda_{p q} \gamma^{p q} \psi_{\bar{p}}+\bar{\Lambda}_{\bar{p} \bar{q}} \psi^{\bar{q}}} \\
& \quad+\frac{1}{32}\left(\frac{1}{5 !} Y^{m n p q r} \gamma_{m n p q r}-14 X_{3}^{p} \gamma_{p}\right)\left(\hat{\mathcal{D}}_{\bar{p}} \rho+\gamma^{A} \mathcal{D}_{A}^{b} \psi_{\bar{p}}\right),
\end{aligned}
$$

where, with

$$
\begin{aligned}
& Y^{m n p q r}=i \bar{\varepsilon}_{2} \gamma^{m n p q r} \varepsilon_{1} \\
& \Phi_{A p q}^{\prime}=\Phi_{A p q}^{0}+i \frac{1}{24} \bar{\rho} \gamma_{A p q} \rho-i \frac{7}{24} \bar{\psi}^{\bar{r}} \gamma_{A p q} \psi_{\bar{r}}
\end{aligned}
$$

the parameters are given by

$$
\begin{aligned}
& X_{3}^{A}=i \bar{\varepsilon}_{2} \gamma^{A} \varepsilon_{1} \\
& \varepsilon_{3}=i \frac{1}{2}\left[\left(\bar{\varepsilon}_{2} \gamma^{p} \varepsilon_{1}\right) \gamma_{p} \rho+\left(\bar{\rho} \varepsilon_{2}\right) \varepsilon_{1}-\left(\bar{\rho} \varepsilon_{1}\right) \varepsilon_{2}\right] \\
& \Lambda_{p q}=2 \hat{\mathcal{D}}_{[p} X_{3 q]}-i \frac{1}{48} \bar{\psi}^{\bar{p}} \gamma^{l m n} \psi_{\bar{p}} Y_{l m n p q}+\Phi_{A p q}^{\prime} X_{3}^{A}, \\
& \bar{\Lambda}_{\bar{p} \bar{q}}=\left(\bar{\Phi}_{A \bar{p} \bar{q}}^{0}-i \bar{\psi}_{\bar{p}} \gamma_{A} \psi_{\bar{q}}\right) X_{3}^{A} .
\end{aligned}
$$

In particular, $\Lambda_{p q}=-\Lambda_{q p}$ and $\bar{\Lambda}_{\bar{p} \bar{q}}=-\bar{\Lambda}_{\bar{q} \bar{p}}$ correspond to the $\mathbf{s o}(1,9)$ and $\mathbf{s o}(9,1)$ local Lorentz symmetry parameters respectively. Further, since $X_{3}^{p} C \gamma_{p}$ and $C\left(\frac{1}{5 !} Y^{m n p q r} \gamma_{m n p q r}-14 Y^{p} \gamma_{p}\right)$ are symmetric, the terms that are proportional to the fermionic equations of motion in (35) correspond to the fermionic "trivial" gauge symmetry [40].

Identifying the common symmetry parameters in 37 on the right hand side of each line in (35) provides a nontrivial consistency check. For this, note also identically,

$$
\delta_{\varepsilon_{3}} d=i \frac{1}{2} \bar{\varepsilon}_{3} \rho=0 .
$$

Therefore, the commutator of DFT supersymmetry transformations (30) closes up to the strong level matching constraint and every gauge symmetry listed in Table I] as well as the fermionic equations of motion,

$$
\left[\delta_{\varepsilon_{1}}, \delta_{\varepsilon_{2}}\right] \equiv \hat{\mathcal{L}}_{X_{3}}+\delta_{\varepsilon_{3}}+\delta_{\text {so }(1,9)}+\delta_{\mathbf{s o}(9,1)}+\delta_{\text {trivial }}
$$

\section{COMMENTS}

From (17), (21), (24), up to the strong level matching constraint (2), we obtain

$$
\begin{gathered}
P^{A B} \bar{P}^{C D} S_{A C B D}=-\frac{1}{2} P^{A B} \bar{P}^{C D} \Gamma_{A B}^{E} \Gamma_{E C D} \equiv 0, \\
P^{A B} P^{C D} S_{A C B D} \equiv P^{A B} S_{A B}, \\
\bar{P}^{A B} \bar{P}^{C D} S_{A C B D} \equiv \bar{P}^{A B} S_{A B} .
\end{gathered}
$$

Hence, the bosonic part of the Lagrangian (28) may reduce to the single term, $\frac{1}{8} \mathcal{H}^{A B} S_{A B}$ that was previously suggested in [15] with the so-called generalized metric, $\mathcal{H}_{A B}=P_{A B}-\bar{P}_{A B}$. However, the expression in (28) appears more directly relevant to the 1.5 formalism (33).

The double-vielbeins, $V_{A p}, \bar{V}_{A \bar{p}}$ (8), admit explicit parametrization in terms of the Kalb-Ramond $B$-field and a pair of zehnbeins corresponding to the common spacetime metric, $e_{\mu}^{p} e_{\nu}^{q} \eta_{p q}=-\bar{e}_{\mu}{ }_{\mu}^{\bar{e}} \bar{e}_{\nu}{ }^{\bar{q}} \bar{\eta}_{\bar{p} \bar{q}}=g_{\mu \nu}$, in an $\mathbf{O}(10,10)$ covariant manner [15, 16]. Gauge fixing the two zehnbeins equal to each other breaks $\mathbf{O}(10,10)$ to $\mathbf{O}(10) \rtimes \mathbf{G L}(10)$ [15] and the pair of local Lorentz symmetries to a single one. Further, as shown in detail in the Appendix, it reduces our supersymmetric DFT to the ten-dimensional $\mathcal{N}=1$ supergravity of eleven-dimensional origin [41] (c.f. [42, 43]). This result seems to suggest that a generic supergravity theory is an $\mathbf{O}(D, D)$ and hence double local Lorentz broken double field theory.

The supersymmetric completion of Refs. 23, 24] for type IIA/IIB supergravity remains as a future work.

Acknowledgements: We wish to thank Neil Copland and Dimitris Tsimpis for useful comments. The work was supported by the National Research Foundation of Korea(NRF) grants funded by the Korea government (MEST) with the Grant No. 2005-0049409 (CQUeST) and Grant No. 2010-0002980. The work by IJ is partially supported by NRF though the KoreaCERN theory collaboration.

Note added: After our submission, Ref. 44] appeared in arXiv which also addresses the supersymmetrization of DFT, yet up to the quadratic order in fermions. It differs in detail from our full order analysis.
[1] T. H. Buscher, Phys. Lett. B 159 (1985) 127.

[2] T. H. Buscher, Phys. Lett. B 194 (1987) 59.

[3] T. H. Buscher, Phys. Lett. B 201 (1988) 466.

[4] A. Giveon, E. Rabinovici and G. Veneziano, Nucl. Phys.
B 322 (1989) 167.

[5] A. A. Tseytlin, Phys. Lett. B 242, 163 (1990).

[6] A. A. Tseytlin, Nucl. Phys. B 350, 395 (1991).

[7] W. Siegel, Phys. Rev. D 47, 5453 (1993). 
[8] W. Siegel, Phys. Rev. D 48, 2826 (1993).

[9] C. Hull and B. Zwiebach, JHEP 0909, 099 (2009).

[10] C. Hull and B. Zwiebach, JHEP 0909, 090 (2009).

[11] O. Hohm, C. Hull and B. Zwiebach, JHEP 1007, 016 (2010). arXiv:1003.5027 [hep-th]].

[12] O. Hohm, C. Hull and B. Zwiebach, JHEP 1008, 008 (2010). arXiv:1006.4823 [hep-th]].

[13] S. K. Kwak, JHEP 1010 (2010) 047.

[14] I. Jeon, K. Lee and J.-H. Park, JHEP 1104 (2011) 014. arXiv:1011.1324 [hep-th]].

[15] I. Jeon, K. Lee, J.-H. Park, Phys. Rev. D84 (2011) 044022. arXiv:1105.6294 [hep-th]].

[16] I. Jeon, K. Lee and J.-H. Park, JHEP 11 (2011) 025. arXiv:1109.2035 [hep-th]].

[17] I. Jeon, K. Lee, J. -H. Park, Phys. Lett. B701 (2011) 260-264. arXiv:1102.0419 [hep-th]].

[18] O. Hohm, S. K. Kwak, J. Phys. A A44 (2011) 085404.

[19] O. Hohm, S. K. Kwak, JHEP 1106 (2011) 096.

[20] D. S. Berman, H. Godazgar, M. J. Perry, Phys. Lett. B700 (2011) 65-67. arXiv:1103.5733 [hep-th]].

[21] D. C. Thompson, JHEP 1108 (2011) 125.

[22] N. B. Copland, Nucl. Phys. B854 (2012) 575-591.

[23] O. Hohm, S. K. Kwak, B. Zwiebach, Phys. Rev. Lett. 107 (2011) 171603. arXiv:1106.5452 [hep-th]].

[24] O. Hohm, S. K. Kwak, B. Zwiebach, JHEP 1109 (2011) 013. arXiv:1107.0008 [hep-th]].

[25] C. Albertsson, S. -H. Dai, P. -W. Kao, F. -L. Lin, JHEP 1109 (2011) 025. arXiv:1107.0876 [hep-th]].

[26] O. Hohm, S. K. Kwak, arXiv:1108.4937 [hep-th]].

[27] N. Kan, K. Kobayashi, K. Shiraishi, arXiv:1108.5795 [hep-th]].
[28] G. Aldazabal, W. Baron, D. Marques, C. Nunez, arXiv:1109.0290 [hep-th]].

[29] D. Geissbuhler, arXiv:1109.4280 [hep-th]].

[30] D. S. Berman, E. T. Musaev, M. J. Perry, arXiv:1110.3097 [hep-th]].

[31] N. B. Copland, arXiv:1111.1828 [hep-th]].

[32] T. Courant, "Dirac Manifolds," Trans. Amer. Math. Soc. 319: 631-661, 1990.

[33] M. Gualtieri, Ph.D. Thesis, Oxford University, 2003. arXiv:math/0401221

[34] M. Grana, R. Minasian, M. Petrini and D. Waldram, JHEP 0904 (2009) 075.

[35] N. Hitchin, Quart. J. Math. Oxford Ser. 54, 281 (2003).

[36] N. Hitchin, arXiv:1008.0973 [math.DG].

[37] A. Coimbra, C. Strickland-Constable, D. Waldram, arXiv:1107.1733 [hep-th]].

[38] D. S. Berman and M. J. Perry, arXiv:1008.1763 [hep-th]].

[39] D. S. Berman, H. Godazgar, M. Godazgar, M. J. Perry, arXiv:1110.3930 [hep-th]].

[40] M. Henneaux, C. Teitelboim, Princeton, USA: Univ. Pr. (1992) $520 \mathrm{p}$.

[41] E. Cremmer, B. Julia and J. Scherk, Phys. Lett. B 76 (1978) 409.

[42] A. H. Chamseddine, Nucl. Phys. B 185 (1981) 403.

[43] E. Bergshoeff, M. de Roo, B. de Wit and P. van Nieuwenhuizen, Nucl. Phys. B 195 (1982) 97.

[44] O. Hohm and S. K. Kwak, arXiv:1111.7293 [hep-th].

[45] A video presentation of this work is available at http://www.newton.ac.uk/programmes/BSM/seminars/021611301.html

\section{Appendix A: Reduction from $11 D$ to $10 D$ supergravity}

Here after spelling out the $11 D$ supergravity by Cremmer, Julia and Scherk 41], we set up our ansatz of its dimensional reduction to the $10 D$ minimal supergravity. Our ansatz differs in detail from those in [42, 43], and is designed to produce the precise $10 D \mathcal{N}=1$ supergravity in string frame with which our supersymmetric DFT matches.

\section{1. $11 D$ supergravity from Ref. [41]}

With the eleven-dimensional curved and flat vector indices, $M, N, P, \cdots$ and $A, B, C, \cdots$ respectively, the $11 D$ supergravity action is

$$
\begin{aligned}
\mathcal{L}_{11 D}= & \frac{E}{4 \kappa^{2}} R(E, \omega)-i \frac{E}{2} \bar{\Psi}_{M} \Gamma^{M N P} D_{N}\left(\frac{\omega+\hat{\omega}}{2}\right) \Psi_{P}-\frac{E}{48} F_{M N P Q} F^{M N P Q} \\
& +i \frac{E \kappa}{192}\left(\bar{\Psi}_{M} \Gamma^{M N P Q R S} \Psi_{N}+12 \bar{\Psi}^{P} \Gamma^{Q R} \Psi^{S}\right)\left(F_{P Q R S}+\hat{F}_{P Q R S}\right) \\
& -\frac{2 \kappa}{(144)^{2}} \epsilon^{M_{1} M_{2} M_{3} M_{4} N_{1} N_{2} N_{3} N_{4} P_{1} P_{2} P_{3}} F_{M_{1} M_{2} M_{3} M_{4} F_{N_{1} N_{2} N_{3} N_{4}} A_{P_{1} P_{2} P_{3}},}
\end{aligned}
$$

where, $D_{M}$ is a covariant derivative with respect to the $11 D$ local Lorentz transformation only, such that with the standard Christoffel symbol, it satisfies

$$
D_{M}(\omega) E_{N}^{A}=\Gamma_{M}{ }^{P}{ }_{N} E_{P}^{A}, \quad \Gamma_{M}{ }^{P}{ }_{N}=\left\{M^{P}{ }_{N}\right\}+h_{M}{ }^{P}{ }_{N} .
$$


Further we have

$$
\begin{aligned}
& \omega_{M A B}=-\omega_{M B A}=\frac{1}{2}\left[E_{A}^{N}\left(\partial_{M} E_{N B}-\partial_{N} E_{M B}+E_{M}{ }^{C} \partial_{P} E_{N C} E_{B}^{P}\right)-(A \leftrightarrow B)\right]+h_{M A B}, \\
& h_{M A B}=-h_{M B A}=i \kappa^{2}\left(\frac{1}{4} \Psi_{P} \Gamma_{M A B}{ }^{P Q} \Psi_{Q}+\bar{\Psi}_{M} \Gamma_{[A} \Psi_{B]}+\frac{1}{2} \bar{\Psi}_{A} \Gamma_{M} \Psi_{B}\right),
\end{aligned}
$$

and

$$
\begin{aligned}
& \hat{\omega}_{M A B}=\omega_{M A B}-i \frac{\kappa^{2}}{4} \bar{\Psi}_{P} \Gamma^{P Q}{ }_{M A B} \Psi_{Q}, \\
& \hat{F}_{M N P Q}=F_{M N P Q}-3 i \kappa \bar{\Psi}_{[M} \Gamma_{N P} \Psi_{Q]} .
\end{aligned}
$$

The supersymmetry transformations are

$$
\begin{aligned}
& \delta_{\mathcal{E}} E^{A}{ }_{M}=i \kappa \overline{\mathcal{E}} \Gamma^{A} \Psi_{M}, \\
& \delta_{\mathcal{E}} \Psi_{M}=\frac{1}{\kappa} \hat{D}_{M}(\hat{\omega}, \hat{F}) \mathcal{E}, \\
& \delta_{\mathcal{E}} A_{M N P}=i \frac{3}{2} \overline{\mathcal{E}} \Gamma_{[M N} \Psi_{P]},
\end{aligned}
$$

where

$$
\hat{D}_{M}(\hat{\omega}, \hat{F})=D_{M}(\hat{\omega})-\frac{\kappa}{144}\left(\Gamma_{M}^{P Q R S}-8 \delta_{M}^{P} \Gamma^{Q R S}\right) \hat{F}_{P Q R S} .
$$

\section{Ansatz of the reduction}

With the decomposition of the curved and the flat $11 D$ vector indices,

$$
M=(\mu, 11), \quad A=(a, z),
$$

after putting $\kappa=1$, our ansatz of the reduction is as follows. For the elfbein we set

$$
E_{M}^{A}=\left(\begin{array}{cc}
e^{-\frac{1}{3} \phi} e_{\mu}{ }^{a} & 0 \\
0 & e^{\frac{2}{3} \phi}
\end{array}\right),
$$

and for the three-form gauge field, we put

$$
A_{\mu \nu \lambda}=0, \quad A_{\mu \nu 11}=\frac{1}{2} B_{\mu \nu} .
$$

Further for the fermions, we write

$$
\begin{aligned}
& \Psi_{a}=\frac{1}{6} 2^{\frac{1}{4}} e^{\frac{1}{6} \phi}\left(5 \psi_{a}-\gamma_{a b} \psi^{b}-\gamma_{a} \rho\right) \\
& \Psi_{z}=-\frac{1}{3} 2^{\frac{1}{4}} e^{\frac{1}{6} \phi}\left(\rho+\gamma^{a} \psi_{a}\right) \\
& \mathcal{E}=2^{-\frac{1}{4}} e^{-\frac{1}{6} \phi} \varepsilon
\end{aligned}
$$

and impose the chirality conditions,

$$
\gamma^{(10)} \psi_{a}=\psi_{a}, \quad \gamma^{(10)} \rho=-\rho . \quad \gamma^{(10)} \varepsilon=\varepsilon .
$$


Finally, we reduce the $11 D$ supersymmetry A5 to $10 D \mathcal{N}=1$ supersymmetry, by adding an so(1,9) local Lorentz transformation, parametrized by $\Lambda_{a b}^{\prime}=i \frac{1}{6} \bar{\varepsilon} \gamma_{a b}\left(\rho+\gamma^{c} \psi_{c}\right)$,

$$
\delta_{\mathcal{E}}^{11 D}+\delta_{\Lambda^{\prime}} \Rightarrow \delta_{\varepsilon}^{10 D} .
$$

\section{3. $10 D \mathcal{N}=1$ supergravity}

The resulting $10 D \mathcal{N}=1$ supergravity action is, after heavy usage of the Fierz identities (A23), A24, (A25), (A26), A27,

$$
\begin{aligned}
\mathcal{L}_{10 D}=e \times e^{-2 \phi}[R & +4 \partial_{\mu} \phi \partial^{\mu} \phi-\frac{1}{12} H_{\lambda \mu \nu} H^{\lambda \mu \nu} \\
& +i 2 \sqrt{2} \bar{\rho} \gamma^{m}\left(\partial_{m} \rho+\frac{1}{4} \omega_{m n p} \gamma^{n p} \rho+\frac{1}{24} H_{m n p} \gamma^{n p} \rho\right) \\
& -i 4 \sqrt{2} \bar{\psi}^{p}\left(\partial_{p} \rho+\frac{1}{4} \omega_{p q r} \gamma^{q r} \rho+\frac{1}{8} H_{p q r} \gamma^{q r} \rho\right) \\
& -i 2 \sqrt{2} \bar{\psi}^{p} \gamma^{m}\left(\partial_{m} \psi_{p}+\frac{1}{4} \omega_{m n p} \gamma^{n p} \psi_{p}+\omega_{m p q} \psi^{q}+\frac{1}{24} H_{m n p} \gamma^{n p} \psi_{p}-\frac{1}{2} H_{m p q} \psi^{q}\right) \\
& \left.+\frac{1}{24}\left(\bar{\psi}^{q} \gamma_{m n p} \psi_{q}\right)\left(\bar{\psi}^{r} \gamma^{m n p} \psi_{r}\right)-\frac{1}{48}\left(\bar{\psi}^{q} \gamma_{m n p} \psi_{q}\right)\left(\bar{\rho} \gamma^{m n p} \rho\right)\right]
\end{aligned}
$$

of which the $\mathcal{N}=1$ supersymmetry is, from (A12), given by

$$
\begin{aligned}
\delta_{\varepsilon} \phi= & i \frac{1}{2} \bar{\varepsilon}\left(\rho+\gamma^{a} \psi_{a}\right), \\
\delta_{\varepsilon} e_{\mu}^{a}= & i \bar{\varepsilon} \gamma^{a} \psi_{\mu}, \\
\delta_{\varepsilon} B_{\mu \nu}= & -2 i \bar{\varepsilon} \gamma_{[\mu} \psi_{\nu]}, \\
\delta_{\varepsilon} \rho=- & \frac{1}{\sqrt{2}} \gamma^{a}\left(\partial_{a} \varepsilon+\frac{1}{4} \omega_{a b c} \gamma^{b c} \varepsilon+\frac{1}{24} H_{a b c} \gamma^{b c} \varepsilon-\partial_{a} \phi \varepsilon\right) \\
& +i \frac{1}{48}\left(\overline{\psi^{d}} \gamma_{a b c} \psi_{d}\right) \gamma^{a b c} \varepsilon+i \frac{1}{192}\left(\bar{\rho} \gamma_{a b c} \rho\right) \gamma^{a b c} \varepsilon \\
& +i \frac{1}{2}\left(\bar{\varepsilon} \gamma_{[a} \psi_{b]}\right) \gamma^{a b} \rho, \\
\delta_{\varepsilon} \psi_{a}= & \frac{1}{\sqrt{2}}\left(\partial_{a} \varepsilon+\frac{1}{4} \omega_{a b c} \gamma^{b c} \varepsilon+\frac{1}{8} H_{a b c} \gamma^{b c} \varepsilon\right) \\
& -i \frac{1}{2}(\bar{\rho} \varepsilon) \psi_{a}-i \frac{1}{4}\left(\bar{\rho} \psi_{a}\right) \varepsilon+i \frac{1}{8}\left(\bar{\rho} \gamma_{b c} \psi_{a}\right) \gamma^{b c} \varepsilon \\
& +i \frac{1}{2}\left(\bar{\varepsilon} \gamma_{[b} \psi_{c]}\right) \gamma^{b c} \psi_{a} .
\end{aligned}
$$

Here we set $\partial_{p}=\left(e^{-1}\right)_{p}{ }^{\mu} \partial_{\mu}$ and assume the standard spin connection, $\omega_{\mu p q}=\left(e^{-1}\right)_{p}{ }^{\nu} \nabla_{\mu} e_{\nu q}$, with the diffeomorphsim covariant derivative, $\nabla_{\mu}$, given by the torsionless, Christoffel symbol.

\section{Matching the supersymmetric DFT and the $10 D$ supergravity}

The double-vielbein satisfies the defining properties (8),

$$
\begin{array}{ll}
V_{A p} V_{q}^{A}=\eta_{p q}, & V_{A p} \bar{V}_{\bar{q}}^{A}=0, \\
\bar{V}_{A \bar{p}} \bar{V}_{\bar{q}}^{A}=\bar{\eta}_{\bar{p} \bar{q}}, & V_{A p} V_{B}{ }^{p}+\bar{V}_{A \bar{p}} \bar{V}_{B} \bar{p}=\mathcal{J}_{A B},
\end{array}
$$


which are manifestly $\mathbf{O}(10,10)$ covariant. Assuming that the upper half blocks of $V_{A P}$ and $\bar{V}_{A \bar{p}}$ are non-degenerate, the double-vielbein takes the following most general form [15, 16]

$$
V_{A p}=\frac{1}{\sqrt{2}}\left(\begin{array}{c}
\left(e^{-1}\right)_{p}{ }^{\mu} \\
(B+e)_{\nu p}
\end{array}\right), \quad \bar{V}_{A \bar{p}}=\frac{1}{\sqrt{2}}\left(\begin{array}{c}
\left(\bar{e}^{-1}\right)_{\bar{p}}{ }^{\mu} \\
(B+\bar{e})_{\nu \bar{p}}
\end{array}\right) .
$$

Here $e_{\mu}{ }^{p}$ and $\bar{e}_{\nu}{ }^{\bar{p}}$ are two copies of the zehnbeins corresponding to the same spacetime metric,

$$
e_{\mu}^{p} e_{\nu}^{q} \eta_{p q}=-\bar{e}_{\mu}{ }^{\bar{p}} \bar{e}_{\nu} \bar{q}_{\bar{\eta}} \bar{\eta}_{\bar{p}}=g_{\mu \nu},
$$

and $B_{\mu \nu}=-B_{\nu \mu}$ can be identified as the Kalb-Ramond two-form gauge field. We also set in A16),

$$
B_{\mu p}=B_{\mu \nu}\left(e^{-1}\right)_{p}{ }^{\nu}, \quad B_{\mu \bar{p}}=B_{\mu \nu}\left(\bar{e}^{-1}\right)_{\bar{p}}{ }^{\nu} .
$$

In particular, $\left(\bar{e}^{-1} e\right)_{\bar{p}}^{p}$ and $\left(e^{-1} \bar{e}\right)_{p}{ }^{\bar{p}}$ are local Lorentz transformations, satisfying

$$
\begin{aligned}
\left(\bar{e}^{-1} e\right)_{\bar{p}}{ }^{p}\left(\bar{e}^{-1} e\right)_{\bar{q}}{ }^{q} \eta_{p q}=-\bar{\eta}_{\bar{p} \bar{q}}, \\
\left(e^{-1} \bar{e}\right)_{p}{ }_{p}\left(e^{-1} \bar{e}\right)_{q} \bar{q}_{\bar{p} \bar{q}}=-\eta_{p q} .
\end{aligned}
$$

Since (A15) is manifestly $\mathbf{O}(10,10)$ covariant and the parametrization (A16) is quite generic, the constraint (A17) is compatible with the $\mathbf{O}(10,10)$ structure. For its explicit verification, we refer (41), (42), (43) in [15] or section 3.2 of [16].

Now, with the explicit parametrization (A16), from (31) in [15] and (4.51) in [16], upon the strong level matching constraint, $\tilde{\partial} \equiv 0$, our supersymmetric DFT Lagrangian (28) decomposes into three parts: genuine bosonic terms, quadratic fermion terms, and quartic fermion terms,

$$
\mathcal{L}_{\mathrm{SDFT}}=\mathcal{L}_{0}+\mathcal{L}_{2}+\mathcal{L}_{4}
$$

each of which reads explicitly,

$$
\begin{aligned}
e^{2 d} \mathcal{L}_{0}= & \frac{1}{8}\left(P^{A B} P^{C D}-\bar{P}^{A B} \bar{P}^{C D}\right) S_{A B C D}^{0} \\
\equiv & \frac{1}{8}\left(R+4 \square \phi-4 \partial_{\mu} \partial^{\mu} \phi-\frac{1}{12} H_{\lambda \mu \nu} H^{\lambda \mu \nu}\right) \\
e^{2 d} \mathcal{L}_{2}= & i \frac{1}{2} \bar{\rho} \gamma^{A} \mathcal{D}_{A}^{0} \rho+i \bar{\psi}^{A} \mathcal{D}_{A}^{0} \rho+i \frac{1}{2} \bar{\psi}^{B} \gamma^{A} \mathcal{D}_{A}^{0} \psi_{B} \\
\equiv & i \frac{1}{2 \sqrt{2}} \bar{\rho} \gamma^{m}\left(\partial_{m} \rho+\frac{1}{4} \omega_{m n p} \gamma^{n p} \rho+\frac{1}{24} H_{m n p} \gamma^{n p} \rho\right)+i \frac{1}{\sqrt{2}} \bar{\psi}^{\bar{p}}\left(\partial_{\bar{p}} \rho+\frac{1}{4} \omega_{\bar{p} q r} \gamma^{q r} \rho+\frac{1}{8} H_{\bar{p} q r} \gamma^{q r} \rho\right) \\
& +i \frac{1}{2 \sqrt{2}} \bar{\psi}^{\bar{p}} \gamma^{m}\left(\partial_{m} \psi_{\bar{p}}+\frac{1}{4} \omega_{m n p} \gamma^{n p} \psi_{\bar{p}}+\bar{\omega}_{m \bar{p} \bar{q}} \psi^{\bar{q}}+\frac{1}{24} H_{m n p} \gamma^{n p} \psi_{\bar{p}}+\frac{1}{2} H_{m \bar{p} \bar{q}} \psi^{\bar{q}}\right) \\
e^{2 d} \mathcal{L}_{4}= & \frac{1}{8}\left[\frac{1}{24}\left(\bar{\psi}^{D} \gamma_{A B C} \psi_{D}\right)\left(\bar{\psi}^{E} \gamma^{A B C} \psi_{E}\right)+\frac{1}{48}\left(\bar{\psi}^{D} \gamma_{A B C} \psi_{D}\right)\left(\bar{\rho} \gamma^{A B C} \rho\right)\right] \\
= & \frac{1}{8}\left[\frac{1}{24}\left(\bar{\psi}^{\bar{p}} \gamma_{m n p} \psi_{\bar{p}}\right)\left(\bar{\psi}^{\bar{q}} \gamma^{m n p} \psi_{\bar{q}}\right)+\frac{1}{48}\left(\bar{\psi}^{\bar{p}} \gamma_{m n p} \psi_{\bar{p}}\right)\left(\bar{\rho} \gamma^{m n p} \rho\right)\right],
\end{aligned}
$$

where $\partial_{p}=\left(e^{-1}\right)_{p}{ }^{\mu} \partial_{\mu}, \partial_{\bar{p}}=\left(\bar{e}^{-1}\right)_{\bar{p}}^{\mu} \partial_{\mu}, \omega_{\mu p q}=\left(e^{-1}\right)_{p}^{\nu} \nabla_{\mu} e_{\nu q}, \bar{\omega}_{\mu \bar{p} \bar{q}}=\left(\bar{e}^{-1}\right)_{\bar{p}}{ }^{\nu} \nabla_{\mu} \bar{e}_{\nu \bar{q}}$, etc.

After gauge fixing, $\bar{e}_{\mu}^{\bar{p}}=e_{\mu}^{p}$, with the identification, $\bar{\eta}_{\bar{p} \bar{q}}=-\eta_{p q}$, which breaks $\mathbf{O}(10,10)$ to $\mathbf{O}(10) \rtimes \mathbf{G L}(10)$ [15], and the pair of local Lorentz symmetries to a single one, $\mathbf{s o}(1,9) \times \mathbf{s o}(9,1) \rightarrow \mathbf{s o}(1,9)$, it is straightforward to check that the supersymmetric DFT Lagrangian (A21) coincides with the $10 D \mathcal{N}=1$ supergravity Lagrangian (A13). Further, the DFT supersymmetry (30) agrees with the $10 D \mathcal{N}=1$ supersymmetry (A14), up an so(1,9) local Lorentz transformation corresponding to the former of so $(1,9) \times \mathbf{s o}(9,1)$ having the parameter,

$$
-i \bar{\varepsilon} \gamma_{p} \psi_{q}+i \bar{\varepsilon} \gamma_{q} \psi_{p} \text {. }
$$




\section{Fierz identities}

Relevant Fierz identities include

$$
\begin{aligned}
& \bar{\rho} \gamma^{p q r} \rho\left(\bar{\rho} \gamma_{p q r}\right)_{\alpha}=0 \\
& \frac{1}{16} \bar{\rho} \gamma^{p q r} \rho \bar{\psi}_{\bar{p}} \gamma_{p q r} \psi^{\bar{p}}=\bar{\rho} \gamma_{p q} \psi_{\bar{p}} \bar{\rho} \gamma^{p q} \psi^{\bar{p}} \\
& -\frac{1}{1728} \bar{\psi}^{m} \gamma^{n p q} \psi_{m} \bar{\rho} \gamma_{n p q} \rho-\frac{19}{864} \bar{\rho} \gamma^{m n p} \rho \bar{\psi}_{m} \gamma_{n} \psi_{p}+\frac{1}{12} \bar{\rho} \psi^{m} \bar{\rho} \gamma_{m n} \psi^{n}-\frac{11}{864} \bar{\rho} \gamma^{m n} \psi^{p} \bar{\rho} \gamma_{m n} \psi_{p} \\
& -\frac{7}{216} \bar{\rho} \gamma^{m n} \psi^{p} \bar{\rho} \gamma_{n p} \psi_{m}-\frac{1}{432} \bar{\rho} \gamma^{m n} \psi^{p} \bar{\rho} \gamma_{m n p q} \psi^{q}-\frac{1}{864} \bar{\rho} \gamma^{m n p q} \psi_{q} \bar{\rho} \gamma_{m n p r} \psi^{r} \\
& -\frac{1}{1728} \bar{\rho} \gamma^{m n p} \rho \bar{\psi}^{q} \gamma_{q r m n p} \psi^{r}-\frac{1}{144} \bar{\rho} \gamma_{m n} \psi^{n} \bar{\rho} \gamma^{m p} \psi_{p}-\frac{7}{864} \bar{\rho} \gamma^{m n p} \rho \bar{\psi}_{n} \gamma_{m p q} \psi^{q}=0 \text {, } \\
& -\frac{1}{12} \bar{\rho} \psi^{m} \bar{\psi}_{m} \gamma^{n} \psi_{n}+\frac{13}{1728} \bar{\psi}^{m} \gamma^{n p q} \psi_{m} \bar{\rho} \gamma_{p q} \psi_{n}+\frac{19}{288} \bar{\psi}^{m} \gamma^{n} \psi^{p} \bar{\rho} \gamma_{m p} \psi_{n}-\frac{1}{16} \bar{\psi}^{m} \gamma^{n} \psi^{p} \bar{\rho} \gamma_{n p} \psi_{m} \\
& -\frac{1}{72} \bar{\psi}^{m} \gamma_{m} \psi^{n} \bar{\rho} \gamma_{n p} \psi^{p}-\frac{1}{1728} \bar{\psi}^{m} \gamma^{n p q} \psi_{m} \bar{\rho} \gamma_{n p q r} \psi^{r}+\frac{25}{864} \bar{\psi}^{m} \gamma^{n} \psi^{p} \bar{\rho} \gamma_{m n p q} \psi^{q} \\
& -\frac{5}{1728} \bar{\rho} \gamma^{m n} \psi^{p} \bar{\psi}^{q} \gamma_{q r p m n} \psi^{r}-\frac{1}{1728} \bar{\rho} \gamma^{m n p q} \psi_{q} \bar{\psi}^{r} \gamma_{r s m n p} \psi^{s}+\frac{13}{432} \bar{\rho} \gamma^{m n} \psi^{p} \bar{\psi}_{m} \gamma_{n p q} \psi^{q} \\
& +\frac{7}{864} \bar{\rho} \gamma^{m n} \psi^{p} \bar{\psi}_{p} \gamma_{m n q} \psi^{q}+\frac{1}{864} \bar{\rho} \gamma^{m n p q} \psi_{q} \bar{\psi}_{n} \gamma_{m p r} \psi^{r}=0 \text {, }
\end{aligned}
$$

and

$$
\begin{aligned}
& -\frac{11}{216} \bar{\psi}^{m} \gamma^{n p q} \psi_{m} \bar{\psi}_{p} \gamma_{n} \psi_{q}+\frac{73}{432} \bar{\psi}^{m} \gamma^{n} \psi^{p} \bar{\psi}_{m} \gamma_{n} \psi_{p}-\frac{71}{432} \bar{\psi}^{m} \gamma^{n} \psi^{p} \bar{\psi}_{n} \gamma_{m} \psi_{p}-\frac{1}{144} \bar{\psi}^{m} \gamma_{n} \psi^{n} \bar{\psi}_{m} \gamma^{p} \psi_{p} \\
& +\frac{1}{108} \bar{\psi}^{m} \gamma^{n} \psi^{p} \bar{\psi}^{q} \gamma_{q r m n p} \psi^{r}-\frac{1}{108} \bar{\psi}^{m} \gamma^{n p q} \psi_{m} \bar{\psi}_{n} \gamma_{p q r} \psi^{r}-\frac{1}{12} \bar{\psi}^{m} \gamma^{n} \psi^{p} \bar{\psi}_{m} \gamma_{n p q} \psi^{q} \\
& +\frac{1}{144} \bar{\psi}^{m} \gamma^{n} \psi^{p} \bar{\psi}_{n} \gamma_{m p q} \psi^{q}+\frac{1}{864} \bar{\psi}_{m} \gamma^{m n p q r} \psi_{n} \bar{\psi}_{p} \gamma_{q r s} \psi^{s}-\frac{1}{432} \bar{\psi}^{m} \gamma^{n p q} \psi_{q} \bar{\psi}_{n} \gamma_{m p r} \psi^{r} \\
& +\frac{1}{216} \bar{\psi}^{m} \gamma^{n p q} \psi_{q} \bar{\psi}_{m} \gamma_{n p r} \psi^{r}-\frac{1}{192} \bar{\psi}^{q} \gamma^{m n p} \psi_{q} \bar{\psi}^{r} \gamma_{m n p} \psi_{r}=0
\end{aligned}
$$

